\title{
Complex impedance and electric modulus studies of magnetic ceramic $\mathrm{Ni}_{0.27} \mathrm{Cu}_{0.10} \mathrm{Zn}_{0.63} \mathrm{Fe}_{2} \mathrm{O}_{4}$
}

\author{
M. BELAL HOSSEN ${ }^{a, *}$, A. K. M. AKTHER HOSSAIN ${ }^{b}$ \\ ${ }^{a}$ Department of Physics, Chittagong University of Engineering and Technology, Chittagong, Bangladesh \\ ${ }^{b}$ Department of Physics, Bangladesh University of Engineering and Technology, Dhaka, Bangladesh
}

Received: January 14, 2015; Revised: March 16, 2015; Accepted: April 08, 2015

(C) The Author(s) 2015. This article is published with open access at Springerlink.com

\begin{abstract}
The electrical properties of $\mathrm{Ni}_{0.27} \mathrm{Cu}_{0.10} \mathrm{Zn}_{0.63} \mathrm{Fe}_{2} \mathrm{O}_{4}$ (NCZF) prepared from auto combustion synthesis of ferrite powders have been studied by impedance and modulus spectroscopy. We studied frequency and temperature dependencies of impedance and electric modulus of NCZF in a wide frequency range $(20 \mathrm{~Hz}-5 \mathrm{MHz})$ at different measuring temperatures $T_{\mathrm{SM}}\left(30-225{ }^{\circ} \mathrm{C}\right)$. The complex impedance spectra clearly showed both grain and grain boundary effects on the electrical properties. The observed impedance spectra indicated that the magnitude of grain boundary resistance $R_{\mathrm{gb}}$ becomes more prominent compared to grain resistance $R_{\mathrm{b}}$ at room temperature, and with the increase in $T_{\mathrm{SM}}, R_{\mathrm{gb}}$ decreases faster than the intrinsic $R_{\mathrm{b}}$. The frequency response of the imaginary part of impedance showed relaxation behavior at every $T_{\mathrm{SM}}$, and the relaxation frequency variation with $T_{\mathrm{SM}}$ appeared to be of Arrhenius nature and the activation energy has been estimated to be $0.37 \mathrm{eV}$. A complex modulus spectrum was used to understand the mechanism of the electrical transport process, which indicated that a non-Debye type of conductivity relaxation characterizes this material.
\end{abstract}

Keywords: ferrimagnetic ceramics; impedance spectroscopy; electric modulus; temperature dependence

\section{Introduction}

Ferrimagnetic ceramics with spinel structure have drawn considerable attention in recent years due to their potential applications in radio frequency coils, transformer cores, rod antennas, and magnetic cores of read-write heads for high-speed digital tape or disk recording, and they are also good candidates for microwave applications [1,2]. Furthermore,

* Corresponding author.

E-mail: belalcuet@gmail.com ferrimagnetic materials with high resistivity and dielectric constant as well as low dielectric loss, high magnetic transition temperature, and good magnetic, thermal, chemical, and structural stabilities are potential candidates for artificial multiferroic composites. Multiferroic materials are the research focus due to their multifunctionality that could lead to potential applications in highly sensitive sensors and actuators as well as multistate memory devices [3-5]. $\mathrm{NiCuZn}$ ferrites come under the umbrella of the soft ferrites and are chemically symbolized as $\mathrm{DT}_{2} \mathrm{O}_{4}$. In general, the cation distribution in spinel lattice has the 
form $\left(\mathrm{D}_{1-x} \mathrm{~T}_{x}\right)\left[\mathrm{D}_{x} \mathrm{~T}_{2-x}\right] \mathrm{O}_{4}^{2-}$, where $\mathrm{D}$ and $\mathrm{T}$ are divalent $\left(\mathrm{Ni}^{2+}, \mathrm{Cu}^{2+}, \mathrm{Zn}^{2+}\right)$ and trivalent (mainly $\mathrm{Fe}^{3+}$ ) ions respectively, and $x$ is called the degree of inversion. The round and square brackets denote the cations located at the center of tetrahedral (A) lattice of oxygen and those at octahedral (B) lattice, respectively [6]. In NiCuZn ferrites, the presence of specific $\mathrm{Cu}$ content shows remarkable electrical properties $[7,8]$.

Low cost, easy processing, and interesting electrical and magnetic properties make polycrystalline ferrites one of the most important materials in use today. As magnetic materials, ferrites cannot be replaced by any other magnetic material because they are relatively inexpensive and stable, and have a wide range of technological applications $[9,10]$. The high electrical resistivity and consequently low eddy currents and dielectric losses make them very important materials. The electrical properties of these materials have been the subject of continuous investigation, which depend upon the preparation condition, amount of doping element, preparation technique, and measuring temperature, etc. The auto combustion synthesis is beneficial for the preparation of bulk samples due to low cost, highly reactive and easy processing steps, and low power consumption [11].

Magnetic ceramics with semiconducting nature as a function of temperature are important not only for their magnetic properties, but also for the studies of electrical properties from both fundamental and applicational points of view. Polycrystalline ferrites are very good dielectric materials and have many technological and industrial applications ranging from radio to microwave frequencies [12]. The electrical properties of magnetic ceramics sintered in air are highly characterized by conducting grains separated by poorly conducting grain boundaries [6]. It is well known that impedance spectroscopy is an important method to study the electrical properties of ferrites, since impedance of the grains can be separated from other impedance sources, such as electrodes and grain boundaries. One of the important factors, which influences the impedance properties of ferrites, is microstructural effect. Two semicircular arcs are often obtained in Cole-Cole plot, when the grain boundary resistance is larger than that of the grain. The distribution of relaxation time, which results in deviation from ideal semicircles, is attributed to various factors, such as disorder in sample and inhomogeneity in microstructure [13]. Many physical properties of polycrystalline ferrites are very sensitive to the measuring temperature $T_{\mathrm{SM}}$. The grain (bulk) and grain boundary formed heterostructures are the two main components that determine resistivity, and depending on $T_{\mathrm{SM}}$, their electrical properties are more intriguing [13]. Thus, the information about the associated electrical parameters of the components on the heterostructures is important in understanding the overall properties of the materials [14].

In the present work, the electrical properties of $\mathrm{NiCuZn}$ ceramic have been investigated by impedance and modulus spectroscopy. The impedance spectroscopy technique enables us to evaluate and separate out the contributions of various components such as grain and grain boundary to overall electrical properties. The electric modulus approach gives insight into the bulk response which can separate local behavior of defects from electrode effect. The aim of this work is to study the bulk and interface phenomena over a wide range of frequencies at selected temperatures in order to get information about the relaxation time and relaxation amplitude of various processes present in the system, when a small perturbation signal is sent to the system. It is worth noting that various physical parameters and characteristic properties that influence the performance of a ceramic material can be obtained from the analysis of complex impedance spectra.

\section{Experimental}

Polycrystalline spinel ferrite with chemical formula $\mathrm{Ni}_{0.27} \mathrm{Cu}_{0.10} \mathrm{Zn}_{0.63} \mathrm{Fe}_{2} \mathrm{O}_{4}$ (NCZF) was prepared from auto combustion synthesis powders. High purity analytical reagent grade raw materials of $\mathrm{Ni}\left(\mathrm{NO}_{3}\right)_{2} \cdot 6 \mathrm{H}_{2} \mathrm{O}, \mathrm{Cu}\left(\mathrm{NO}_{3}\right)_{2} \cdot 3 \mathrm{H}_{2} \mathrm{O}, \quad \mathrm{Zn}\left(\mathrm{NO}_{3}\right)_{2} \cdot 6 \mathrm{H}_{2} \mathrm{O}$, and $\mathrm{Fe}\left(\mathrm{NO}_{3}\right)_{3} \cdot 9 \mathrm{H}_{2} \mathrm{O}$ were mixed together according to their required stoichiometry. These ingredients were first mixed using minimum amount of ethanol thoroughly by a magnetic stirrer at $70{ }^{\circ} \mathrm{C}$. The mixed solution was then evaporated on a constant temperature water bath. After boiling and ignition of the mixture, a spinel residue was obtained in a few minutes. These powders were crushed and grounded thoroughly. The mixture of the composition was ground to very fine powders and calcined at $700{ }^{\circ} \mathrm{C}$ for $5 \mathrm{~h}$. Finally, the samples were pressed into disk shaped pellets and sintered at $1300{ }^{\circ} \mathrm{C}$ for $5 \mathrm{~h}$. At the start and end of each heat treatment, the samples were allowed to warm and 
cool slowly to room temperature at a rate of $10{ }^{\circ} \mathrm{C} / \mathrm{min}$ and $5{ }^{\circ} \mathrm{C} / \mathrm{min}$, respectively. The phase formation of the desired compound was verified by $\mathrm{X}$-ray diffraction (XRD) measurements. Surface morphology and microstructure of the samples were studied using a high resolution scanning electron microscope (SEM) at room temperature. The pellets were polished to make the opposing surfaces as parallel as possible. Silver paste coating was applied on the opposite faces of pellets, in order to make parallel plate capacitor geometry and the ferrite material as dielectric medium. The complex impedance measurements were carried out using a computer controlled impedance analyzer (Wayne kerr, Model No. 6500B) as functions of frequency $(20 \mathrm{~Hz}-5 \mathrm{MHz})$ and temperature (30$\left.225^{\circ} \mathrm{C}\right)$.

\section{Results and discussion}

\section{1 Structural and microstructural properties}

Figure 1(a) shows the XRD pattern of sintered sample of NCZF at room temperature. From the XRD pattern, it is observed well-defined peaks which correspond to spinel phase of NCZF [8]. No secondary phase peaks are observed, indicating the high quality and single
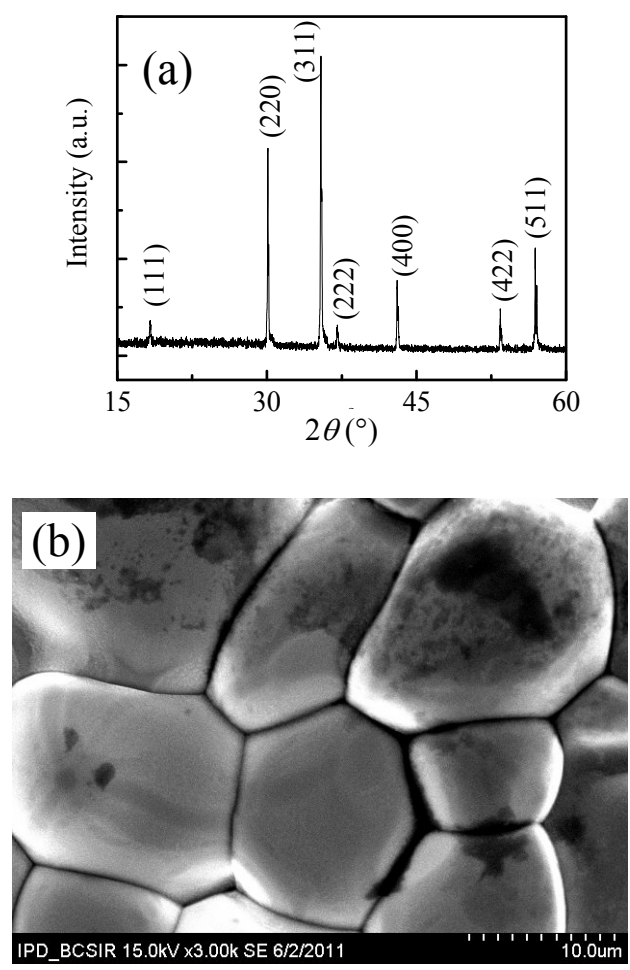

Fig. 1 (a) XRD pattern and (b) SEM micrograph of NCZF at room temperature. phase of the synthesized sample.

Figure 1(b) shows the SEM image of NCZF sintered pellet. As can be seen from the micrograph, the grain growth process is more or less completed during the sintering process. The overall microstructure shows well defined grains with distinct grain boundaries, and the grains are also densely packed without pores or cracks. SEM micrograph reveals the polycrystalline nature of the microstructure with grains of different shapes and sizes ranging between 10 and $20 \mu \mathrm{m}$, which are homogenously distributed throughout the sample surface.

\section{2 Complex impedance analysis}

Complex impedance spectroscopy technique [15] has been used to analyze the electrical properties of a polycrystalline sample and its interface with electronically conducting electrodes in a wide frequency range $(20 \mathrm{~Hz}-5 \mathrm{MHz})$ at different temperatures $\left(30-225{ }^{\circ} \mathrm{C}\right)$. The electrical properties of a material can be represented in terms of complex dielectric permittivity $\varepsilon^{*}$, complex impedance $Z^{*}$, and electric modulus $M^{*}$, which are related to each other as $Z^{*}=Z^{\prime}-\mathrm{j} Z^{\prime \prime}, M^{*}=1 / \varepsilon^{*}(\omega)=\mathrm{j}\left(\omega C_{0}\right) Z^{*}=$ $M^{\prime}+\mathrm{j} M^{\prime \prime}$, where $\left(Z^{\prime}, M^{\prime}\right)$ and $\left(Z^{\prime \prime}, M^{\prime \prime}\right)$ are the real and imaginary components of impedance and modulus, respectively; $j=\sqrt{-1}$ is the imaginary factor; and $\omega$ is the angular frequency, $\omega=2 \pi f$.

The variations of $Z^{\prime}$ and $Z^{\prime \prime}$ and $Z^{\prime}$ versus $Z^{\prime \prime}$ of impedance at selected $T_{\mathrm{SM}}$ are shown in Figs. 2-5. Figure 2 shows the variation of $Z^{\prime}$ with frequency at various $T_{\mathrm{SM}}\left(30-225{ }^{\circ} \mathrm{C}\right)$. It is found that with increase in both temperature and frequency, the value of $Z^{\prime}$ decreases gradually. The pattern of variation of $Z^{\prime}$ shows a low frequency plateau (region I) followed

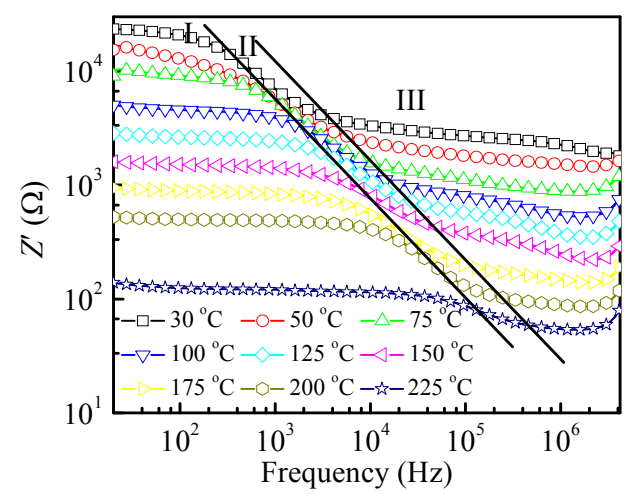

Fig. 2 Variation of $Z^{\prime}$ with frequency at various $T_{\mathrm{SM}}$ for NCZF. 
by a dispersion region II, and, finally, all the curves (region III) show similar pattern of region I with the lower value of $Z^{\prime}$ than region $\mathrm{I}$. The frequency range of region I and region III varies with $T_{\mathrm{SM}}$, and with increasing $T_{\mathrm{SM}}$, frequency range of region $\mathrm{I}$ increases and that of region III decreases; both regions indicate that $Z^{\prime}$ is almost independent of frequency. This behavior indicates an increase in conduction with temperature and frequency. The lower value of $Z^{\prime}$ at region III at various $T_{\mathrm{SM}}$ indicates a possible release of space charge and a consequent lowering of the energy barrier properties [16-18]. At higher frequency, the space charge has less time to relax and the recombination would be faster [19].

Figure 3 illustrates the frequency response of $Z^{\prime \prime}$ at various $T_{\mathrm{SM}}$. This plot is suitable for evaluation of the relaxation frequency of the most resistive component. It is observed that one peak appears for all $T_{\mathrm{SM}}$, indicating the presence of single dielectric relaxation process at the least. The frequency spectrum of $Z^{\prime \prime}$ presents some important features: (i) the appearance of a peak (shown by the line in the plot) at a particular frequency (familiar as relaxation frequency) and gradual decrease in $Z^{\prime \prime}$ to a minimum value with a further rise in frequency and then a tendency to form a second peak; (ii) the decrease in the absolute value of $Z^{\prime \prime}$ with a clear shift of the peak toward higher frequency with the increase of $T_{\mathrm{SM}}$; (iii) the first peak showing the typical peak broadening and the second peak showing the typical peak symmetry. The peak location gives the relaxation time according to the relation $2 \pi f_{\max } \tau=1$, where $f_{\max }$ is the frequency at the maximum of $Z^{\prime \prime}$ versus frequency pattern and $\tau$

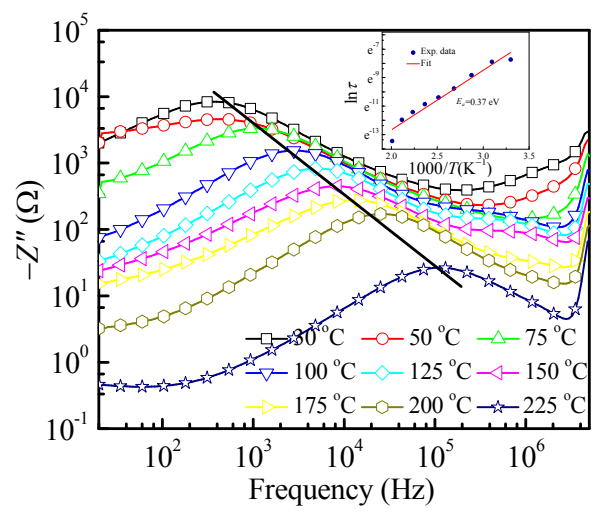

Fig. 3 Variation of $Z^{\prime \prime}$ with frequency and (inset) variation of relaxation time $\tau$ as a function of temperature and the best fit with Arrhenius relation $\tau=\tau_{0} \exp \left[-E_{\mathrm{a}} /\left(k_{\mathrm{B}} T\right)\right]$ for NCZF. is the relaxation time. We have estimated the relaxation frequency and time from the peak position of $Z^{\prime \prime}$ with frequency plots at different $T_{\mathrm{SM}}$. The $\ln \tau$ versus $1 / T$ plot for the sample shows linear behavior (inset of Fig. 3). The typical variation appears to be of Arrhenius nature governed by the relation $\tau=\tau_{0} \exp \left[-E_{\mathrm{a}} /\left(k_{\mathrm{B}} T\right)\right]$, where $k_{\mathrm{B}}$ is the Boltzmann constant; $\tau_{0}$ is the pre-exponential factor; and $E_{\mathrm{a}}$ is the activation energy $[15,16]$. From the Arrhenius fit, the activation energy for the sample has been estimated to be $0.37 \mathrm{eV}$. Broadening of the $Z^{\prime \prime}$ peak with the rise in temperature indicates a non-unique (i.e., multiple) relaxation time scale, whose variance/ distribution is broadening at the higher frequency side after the position of the peak maximum $[18,19]$.

Figure 4 shows the normalized plots of $Z^{\prime \prime}$ with increasing frequency. $Z^{\prime \prime}$ plots are broader than 1.414 decades indicating the deviation from the ideal Debye behavior [20]. Since there is a systematic shift in the peak frequency with $T_{\mathrm{SM}}$ (Fig. 4), it further emphasizes the possibility of the presence of multiple equilibrium states with a distribution of relaxation time and simultaneously a decrease in relaxation time. The shapes of the $Z^{\prime \prime}$ plots obtained at different temperatures remain the same; hence the distribution of the relaxation time is independent of temperature. Relaxation time is decreased with increasing temperature as the dissipated thermal energy assists formed dipoles to follow the motion of the alternating field.

The variation of $Z^{\prime}$ versus $Z^{\prime \prime}$ at different $T_{\mathrm{SM}}$ $\left(30-225{ }^{\circ} \mathrm{C}\right)$ is shown in Fig. 5, which is characterized by the appearance of two semicircles (the first one

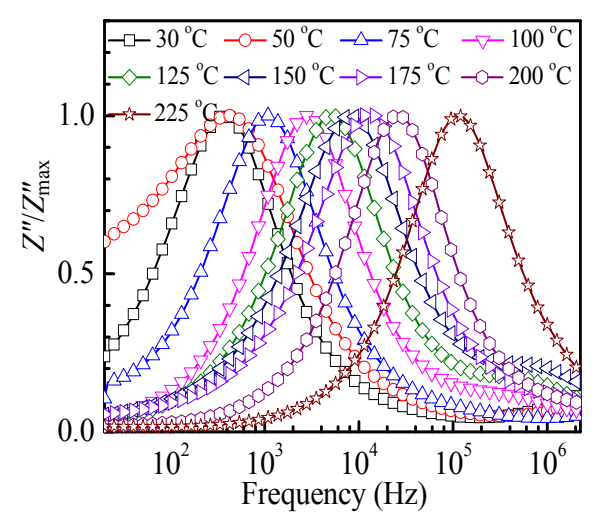

Fig. 4 Variation of normalized reactive part of impedance $Z^{\prime \prime} / Z_{\max }^{\prime \prime}$ with frequency at various $T_{\mathrm{SM}}$ for NCZF. 

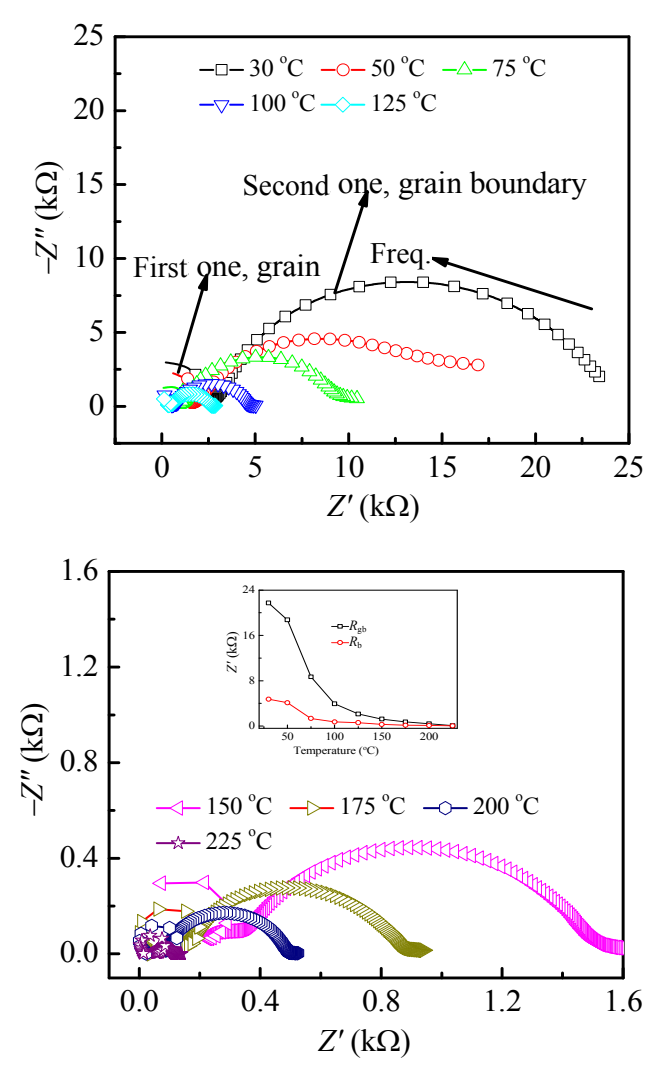

Fig. 5 Variation of $Z^{\prime}$ versus $Z^{\prime \prime}$ at various $T_{\mathrm{SM}}$ for NCZF.

tending to form and the second one forming fully in the measured frequency range) for all $T_{\mathrm{SM}}$. Generally, complex impedance plot typically comprises of two overlapping semicircular arcs at low temperature with center below the real axis, suggesting the departure from ideal Debye behavior [5]. The diameter of the two semicircular arcs is gradually diminished with increase in $T_{\mathrm{SM}}$. The observed two overlapping semicircular arcs are due to the contribution of the intrinsic grain (bulk) and grain boundary electrical properties of the material, and the overlapping condition depends on the difference of grain and grain boundary relaxation time constants. An equivalent circuit can be used in impedance spectroscopy analysis to establish the structure-property relationship of the material. The electrical parameters $\left(R_{\mathrm{b}}\right.$ and $\left.R_{\mathrm{gb}}\right)$ obtained from the fitting using the equivalent circuit models at different temperatures for NCZF have been summarized in Table 1 . The assignment of the two semicircular arcs to the electrical response due to grain interior and grain boundary is consistent with a brick-layer model for a polycrystalline sample [21]. It is observed that both $R_{\mathrm{b}}$ and $R_{\mathrm{gb}}$ decrease with increasing temperature [18]. At all $T_{\mathrm{SM}}$, these two depressed semicircles are observed, centered at the low and high frequency regions. The capacitances for grain and grain boundary are obtained from the maximum height of the semicircles. A relaxation frequency is observed for each contribution (Fig. 4); at this frequency, the condition $Z^{\prime}=-Z^{\prime \prime}$ occurs and it is possible to calculate the corresponding value of equivalent capacitances using the relation $C=$ $1 /(\omega R)$, where $\omega$ is the angular frequency, i.e., $C_{\mathrm{b}}=1 /\left(\omega R_{\mathrm{b}}\right)$ and $C_{\mathrm{gb}}=1 /\left(\omega R_{\mathrm{gb}}\right)$ give the value of grain and grain boundary capacitances respectively as summarized in Table 1. The impedance spectra present depressed semicircles in the low frequency region due to dielectric polarization at the grain boundary/ interface, which is known as the Maxwell-Wagner effect [5].

The diameter of both semicircles decreases with the increase of $T_{\mathrm{SM}}$. This behavior indicates a thermally activated conduction mechanism. In this type of materials (ceramic materials), these semicircles in the impedance plots are generally ascribed to grain (bulk) and grain boundary effects [22]. These two semicircles can be represented by an equivalent circuit constituted by the parallel combinations of resistance $R$ and capacitance $C$, connected in series with another RC parallel connection. One branch is related with sample intrinsic characteristics, i.e., with the grain and other with the interfaces, grain boundaries, and surfaceelectrodes $[14,23,24]$.

In the impedance plots, the relative position of the semicircles depends upon the resistance and capacitance values. The resistance and capacitance of the interfacial grain boundary are usually larger than those of the grain. Thus, the semicircle at high

Table 1 Values of grain and grain boundary resistance and capacitance at various $T_{\mathrm{SM}}$ for NCZF

\begin{tabular}{ccccc}
\hline$T_{\mathrm{SM}}\left({ }^{\circ} \mathrm{C}\right)$ & $R_{\mathrm{gb}}(\Omega)$ & $R_{\mathrm{b}}(\Omega)$ & $C_{\mathrm{gb}}(\mathrm{nF})$ & $C_{\mathrm{b}}(\mathrm{pF})$ \\
\hline 30 & 21750 & 4750 & 20.09 & 6.96 \\
50 & 18750 & 4130 & 19.93 & 8.00 \\
75 & 8700 & 1350 & 16.76 & 24.5 \\
100 & 3950 & 750 & 14.40 & 44.1 \\
125 & 2125 & 600 & 14.41 & 55.1 \\
150 & 1230 & 285 & 14.42 & 108.2 \\
175 & 750 & 160 & 14.61 & 191.3 \\
200 & 430 & 110 & 14.72 & 278.4 \\
225 & 70 & 44 & 20.37 & 694.1 \\
\hline
\end{tabular}


frequency corresponds to the grain (bulk) effect and the second semicircle at lower frequency due to the grain boundary. It is important to remember that the impedance spectra are reproducible within the experimental error. This fact excludes the possibility that the lower frequency loop might be due to other sources such as the contacts between the electrodes and material surfaces.

\section{3 Electric modulus analysis}

Complex electric modulus $\left(M^{*}\right)$ analysis has been adopted to study the frequency and temperature dependence of the conductivity of ceramics [25]. The electric modulus approach gives insight into the bulk response which can separate local behavior of defects from electrode effect [26]. In general, electric modulus corresponds to the relaxation of the electric field in the material when the electric displacement remains constant. The effectiveness of the modulus illustration in the analysis of the relaxation properties has been demonstrated for many polycrystalline ceramics [27-29].

Figure 6 shows the frequency dependence of $M^{\prime}$ at various $T_{\mathrm{SM}}$. It is observed that the value of $M^{\prime}$ is very low (approaching zero) in the low frequency region. A continuous increase in the $M^{\prime}$ dispersion with the increase of frequency shows a tendency to saturate at a maximum asymptotic value for every $T_{\mathrm{SM}}$. The tendency of saturation supports the suggestion that the short range mobility of charge carriers is a conduction process [30]. These interpretations may possibly be related to a lack of restoring force leading the mobility of charge carriers under the action of an induced electric field. Low value of $M^{\prime}$ in the low

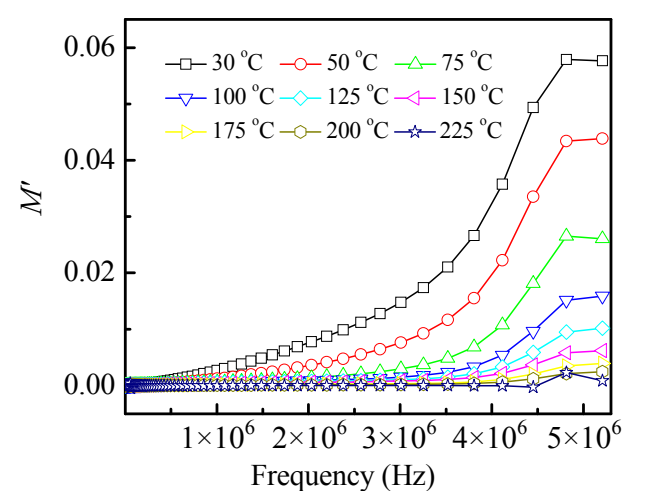

Fig. 6 Variation of $M^{\prime}$ with frequency at various $T_{\mathrm{SM}}$ for NCZF. frequency side supports the conduction phenomena due to long range mobility of charge carriers as well as that the electrode polarization makes a negligible contribution in the material [31].

In the conduction process, the contribution of the holes is smaller than that of the electrons due to their lower mobility. Therefore, the electron exchange between the $\mathrm{Fe}^{2+}$ and $\mathrm{Fe}^{3+}$ ions, which results in local displacements in the direction of the applied external electric field (while the hole exchange between $\mathrm{Ni}^{3+}$ and $\mathrm{Ni}^{2+}$ and $\mathrm{Cu}^{+}$and $\mathrm{Cu}^{2+}$ is in the opposite direction), causes the dielectric polarization in ferrites [32]. Nevertheless, a charge exchange between $\mathrm{Ni}^{2+}$ and $\mathrm{Fe}^{3+}$ can also exist [23]. However the polarization of $\mathrm{Fe}^{2+} \Leftrightarrow \mathrm{Fe}^{3+}$ is the easiest and thus their number will be reflected in the dielectric constant value. The results of Fig. 6 indicate that this number should be the same for all measurements. This is true if we assume that the maximum measurement temperature used $\left(225^{\circ} \mathrm{C}\right)$ is not enough to promote the modification of the oxidation state of this ion. The shapes of the modulus spectroscopic plots obtained at different temperatures remain the same; hence the distribution of the relaxation time is independent of temperature.

The frequency dependence of $M^{\prime \prime}$ at various $T_{\mathrm{SM}}$ is shown in Fig. 7. It is noticed from the figure that $M^{\prime \prime}$ increases with increase in frequency except appearance of a small and broad kink, which is found to be shifted towards the high frequency side with increase in $T_{\mathrm{SM}}$. The frequency region below the peak maximum determines the range in which charge carriers take place due to long range hopping. At the frequency above peak maximum (higher frequency), the carriers are confined to potential wells, being mobile over short distance. The region where the peak

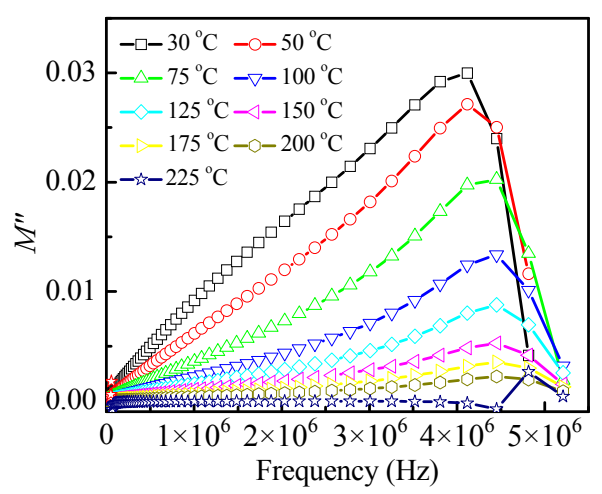

Fig. 7 Variation of $M^{\prime \prime}$ with frequency at various $T_{\mathrm{SM}}$ for NCZF. 
occurs is indicative of the transition from long range to short range mobility with increase in frequency. This type of behavior suggests the existence of a temperature dependent hopping mechanism for electrical conduction (charge transport). The broadening of the peak shows the spread of the relaxation with a relaxation time constant distribution. Furthermore, the appearance of peaks in $M^{\prime \prime}$ provides a clear indication of the real dielectric relaxation process [32-36].

Figure 8 shows the complex modulus plots ( $M^{\prime \prime}$ versus $M^{\prime}$ ) at various $T_{\mathrm{SM}}$. It is clear observed two well resolved semicircles: the first semicircle in the low frequency region indicates the capacitive grain boundary effect (inset of Fig. 8), and the second one represents the capacitive grain effect at higher frequency side except at $T_{\mathrm{SM}}=225^{\circ} \mathrm{C}$.

The common characteristics of Fig. 8 which we observe at various $T_{\mathrm{SM}}$ are summarized as follows: (i) grain boundary and grain effects at lower and higher frequencies respectively; (ii) well resolved big semicircle from grain effect at higher frequencies which decreases with the increase of $T_{\mathrm{SM}}$, while
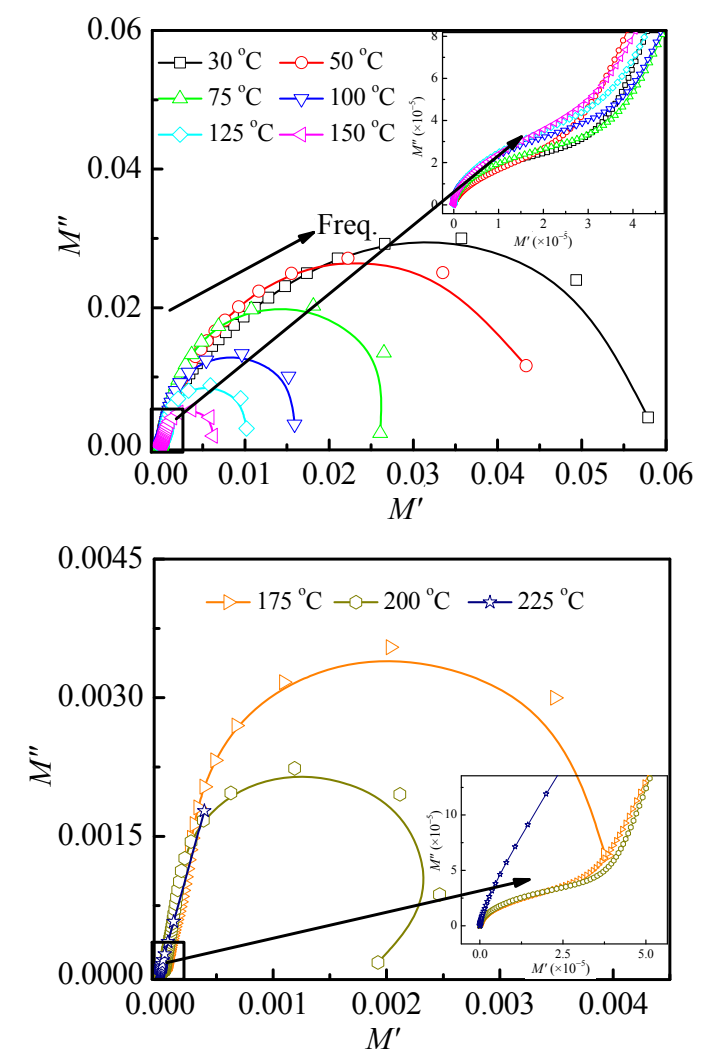

Fig. 8 Variation of $M^{\prime \prime}$ versus $M^{\prime}$ at various $T_{\mathrm{SM}}$ for NCZF. smaller semicircle from grain boundary effect at lower frequencies shows increasing trend; (iii) the magnitude of grain capacitance increases with increasing $T_{\mathrm{SM}}$, while the magnitude of grain boundary capacitance shows decreasing trend; (iv) grain capacitance is strongly temperature dependent, while the grain boundary capacitance shows weakly dependence; (v) at $T_{\mathrm{SM}}=225^{\circ} \mathrm{C}$, only the grain capacitance contribution is observed; (vi) it is also observed that with the increase in $T_{\mathrm{SM}}$, intercept of the grain semicircle on $M^{\prime}$ axis shifts towards the lower values of $M^{\prime}$ which indicates the increase in capacitance. It supports the negative temperature coefficient of resistance (NTCR) type behavior of the material since $C_{\mathrm{b}}$ is inversely proportional to $R_{\mathrm{b}}$.

The double semicircles in Fig. 8 show the dominant grain contribution, whereas the double semicircles in Fig. 5 suggest the dominant grain boundary contribution. It is based on the fact that the impedance plot highlights the phenomenon with the largest resistance, whereas modulus plot picks out those of the smallest capacitance. Complex modulus analysis is suitable when materials have nearly similar resistance but different capacitance [37].

Figure 9 shows the $Z^{\prime \prime}$ and $M^{\prime \prime}$ versus frequency combined plots for $\mathrm{NCZF}$ at $T_{\mathrm{SM}}=100{ }^{\circ} \mathrm{C}$. Similar combined pattern shows all other $T_{\mathrm{SM}}$, but as the measuring temperature increases the difference between two peak frequencies decreases. For Debye type relaxation, the peaks obtained from the $Z^{\prime \prime}$ and $M^{\prime \prime}$ versus frequency plots should coincide [38]. It is observed from Fig. 9 that the $Z^{\prime \prime}$ and $M^{\prime \prime}$ peaks do not overlap, which indicates a non-Debye type relaxation process for the material. The $Z^{\prime \prime}$ and $M^{\prime \prime}$

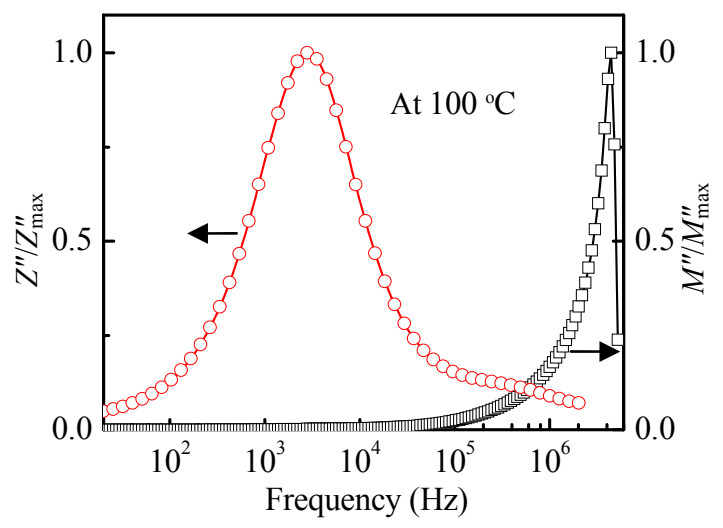

Fig. 9 Variation of $Z^{\prime \prime}$ and $M^{\prime \prime}$ with frequency at $100{ }^{\circ} \mathrm{C}$ for NCZF. 
versus frequency combined plots are used to identify whether the relaxation process in the material is the result of long range or short range movement of charge carriers [39].

\section{Conclusions}

This work reports the results of our investigation on impedance spectra and electrical modulus properties of the magnetic ceramic compound $\mathrm{Ni}_{0.27} \mathrm{Cu}_{0.10} \mathrm{Zn}_{0.63} \mathrm{Fe}_{2} \mathrm{O}_{4}$. Single arc with double semicircles (or its tendency) obtained at a particular temperature corresponding to NCZF composition in both the complex impedance and electric modulus plots suggests the single phase character of the material. We observe two separate conduction processes in impedance and modulus spectra attributed to grain and grain boundary effects. The complex impedance plots reveal two semicircles described by the intrinsic grain (bulk) and grain boundary (interface) effects. The values of $R_{\mathrm{b}}$ and $R_{\mathrm{gb}}$ decrease with increase in temperature and the rate of $R_{\mathrm{gb}}$ decreasing is faster than that of $R_{\mathrm{b}}$ which shows NTCR behavior. The modulus plot shows two well resolved semicircles-smaller one for grain boundary capacitance is weakly dependent on temperature, while large semicircle for grain capacitance is strongly dependent on temperature. The interfacial polarization is a consequence of inhomogeneities in the material, which is dominated by grain boundaries.

Open Access: This article is distributed under the terms of the Creative Commons Attribution License which permits any use, distribution, and reproduction in any medium, provided the original author(s) and the source are credited.

\section{References}

[1] Chen Q, Du P, Huang W, et al. Ferrite with extraordinary electric and dielectric properties prepared from self-combustion technique. Appl Phys Lett 2007, 90: 132907.

[2] Sugimoto M. The past, present, and future of ferrites. $J \mathrm{Am}$ Ceram Soc 1999, 82: 269-280.

[3] Scott JF. Applications of modern ferroelectrics. Science 2007, 315: 954-959.

[4] Chaouchi A, Marinel S, Aliouat M, et al. Low temperature sintering of $\mathrm{ZnTiO}_{3} / \mathrm{TiO}_{2}$ based dielectric with controlled temperature coefficient. J Eur Ceram Soc 2007, 27: 2561-2566.

[5] Lee Y-C, Chiang C-S, Huang Y-L. Microwave dielectric properties and microstructures of $\mathrm{Nb}_{2} \mathrm{O}_{5}-\mathrm{Zn}_{0.95} \mathrm{Mg}_{0.05}$ $\mathrm{TiO}_{3}+0.25 \mathrm{TiO}_{2}$ ceramics with $\mathrm{Bi}_{2} \mathrm{O}_{3}$ addition. $J$ Eur Ceram Soc 2010, 30: 963-970.

[6] Valenzuela R. Magnetic Ceramics. Edinburgh: Cambridge University Press, 1994.

[7] Nam, JH, Jung HH, Shin JY, et al. The effect of $\mathrm{Cu}$ substitution on the electrical and magnetic properties of NiZn ferrites. IEEE T Magn 1995, 31: 3985-3987.

[8] Hossain AKMA, Rahman ML. Enhancement of microstructure and initial permeability due to $\mathrm{Cu}$ substitution in $\mathrm{Ni}_{0.50-x} \mathrm{Cu}_{x} \mathrm{Zn}_{0.50} \mathrm{Fe}_{2} \mathrm{O}_{4}$ ferrites. $J$ Magn Magn Mater 2011, 323: 1954-1962.

[9] Kulikowski J. Soft magnetic ferrites-Development or stagnation? J Magn Magn Mater 1984, 41: 56-62.

[10] Jones Jr. RE, Maniar PD, Moazzami R, et al. Ferroelectric non-volatile memories for low-voltage, low-power applications. Thin Solid Films 1995, 270: 584-588.

[11] Aruna ST, Mukasyan AS. Combustion synthesis and nanomaterials. Curr Opin Solid St M 2008, 12: 44-50.

[12] Verma K, Kumar A, Varshney D. Dielectric relaxation behavior of $\mathrm{A}_{x} \mathrm{Co}_{1-x} \mathrm{Fe}_{2} \mathrm{O}_{4}(\mathrm{~A}=\mathrm{Zn}, \mathrm{Mg})$ mixed ferrites. J Alloys Compd 2012, 526: 91-97.

[13] Costa MM, Júnior PGFM, Sombra ASB. Dielectric and impedance properties' studies of the lead doped $(\mathrm{PbO})-\mathrm{Co}_{2} \mathrm{Y}$ type hexaferrite $\left(\mathrm{Ba}_{2} \mathrm{Co}_{2} \mathrm{Fe}_{12} \mathrm{O}_{22}\left(\mathrm{Co}_{2} \mathrm{Y}\right)\right)$. Mater Chem Phys 2010, 123: 35-39.

[14] Abdullah MH, Yusoff AN. Complex impedance and dielectric properties of an $\mathrm{Mg}-\mathrm{Zn}$ ferrite. $J$ Alloys Compd 1996, 233: 129-135.

[15] MacDonald JR. Impedance Spectroscopy. New York: Wiley, 1987.

[16] Kumar A, Singh BP, Choudhary RNP, et al. Characterization of electrical properties of $\mathrm{Pb}$-modified $\mathrm{BaSnO}_{3}$ using impedance spectroscopy. Mater Chem Phys 2006, 99: 150-159.

[17] Behera $B$, Nayak $P$, Choudhary RNP. Impedance spectroscopy study of $\mathrm{NaBa}_{2} \mathrm{~V}_{5} \mathrm{O}_{15}$ ceramic. $J$ Alloys Compd 2007, 436: 226-232.

[18] Płcharski J, Weiczorek W. PEO based composite solid electrolyte containing nasicon. Solid State Ionics 1988, 28-30: 979-982.

[19] Nobre MAL, Lanfredi S. Dielectric properties of $\mathrm{Bi}_{3} \mathrm{Zn}_{2} \mathrm{Sb}_{3} \mathrm{O}_{14}$ ceramics at high temperature. Mater Lett 2001, 47: 362-366.

[20] Jonscher AK. The 'universal' dielectric response. Nature 1977, 267: 673-679.

[21] Ortega N, Kumar A, Bhattacharya $\mathrm{P}$, et al. Impedance spectroscopy of multiferroic $\mathrm{PbZr}_{x} \mathrm{Ti}_{1-x} \mathrm{O}_{3} / \mathrm{CoFe}_{2} \mathrm{O}_{4}$ layered thin films. Phys Rev B 2008, 77: 014111.

[22] Jamnik J, Maier J. Generalised equivalent circuits for mass and charge transport: Chemical capacitance and its implications. Phys Chem Phys 2001, 3: 1668-1678.

[23] Rathan SV, Govindaraj G. Electrical relaxation studies on $\mathrm{Na}_{2} \mathrm{NbMP}_{3} \mathrm{O}_{12}(\mathrm{M}=\mathrm{Zn}, \mathrm{Cd}, \mathrm{Pb}$ and $\mathrm{Cu})$ phosphate glasses. 
Mater Chem Phys 2010, 120: 255-262.

[24] Peláiz-Barranco A, Gutiérrez-Amador MP, Huanosta A, et al. Phase transitions in ferrimagnetic and ferroelectric ceramics by AC measurements. Appl Phys Lett 1998, 73: 2039.

[25] Roling B. Scaling properties of the conductivity spectra of glasses and supercooled melts. Solid State Ionics 1998, 105: 185-193.

[26] McCrum NG, Read BE, Williams G. An Elastic and Dielectric Effects in Polymeric Solids. New York: Wiley, 1967.

[27] Liu J, Duan C-G, Yin W-G, et al. Dielectric permittivity and electric modulus in $\mathrm{Bi}_{2} \mathrm{Ti}_{4} \mathrm{O}_{11}$. J Chem Phys 2003, 119: 2812.

[28] León C, Lucía ML, Santamaría J. Correlated ion hopping in single-crystal yttria-stabilized zirconia. Phys Rev B 1997, 55: 882 .

[29] Richert R, Wagner H. The dielectric modulus: Relaxation versus retardation. Solid State Ionics 1998, 105: 167-173.

[30] Padmasree KP, Kanchan DK, Kulkami AR. Impedance and modulus studies of the solid electrolyte system $20 \mathrm{CdI}_{2}-80\left[x \mathrm{Ag}_{2} \mathrm{O}-y\left(0.7 \mathrm{~V}_{2} \mathrm{O}_{5}-0.3 \mathrm{~B}_{2} \mathrm{O}_{3}\right)\right]$, where $1 \leqslant x / y$ $\leqslant 3$. Solid State Ionics 2006, 177: 475-482.

[31] Chowdari BVR, Gopalkrishnnan R. AC conductivity analysis of glassy silver iodomolybdate system. Solid State Ionics 1987, 23: 225-233.

[32] Barik SK, Mahapatra PK, Choudhary RNP. Structural and electrical properties of $\mathrm{Na}_{1 / 2} \mathrm{La}_{1 / 2} \mathrm{TiO}_{3}$ ceramics. Appl Phys A 2006, 85: 199-203.
[33] Hou Z-L Cao M-S, Yuan J, et al. High-temperature conductance loss dominated defect level in h-BN: Experiments and first principles calculations. $J$ Appl Phys 2009, 105: 076103.

[34] Song W-L, Cao M-S, Hou Z-L, et al. High dielectric loss and its monotonic dependence of conducting-dominated multiwalled carbon nanotubes/silica nanocomposite on temperature ranging from 373 to $873 \mathrm{~K}$ in $X$-band. Appl Phys Lett 2009, 94: 233110.

[35] Cao M-S, Hou Z-L, Yuan J, et al. Low dielectric loss and non-Debye relaxation of gamma- $\mathrm{Y}_{2} \mathrm{Si}_{2} \mathrm{O}_{7}$ ceramic at elevated temperature in $X$-band. $J$ Appl Phys 2009, 105: 106102.

[36] Cao M-S, Song W-L, Hou Z-L, et al. The effects of temperature and frequency on the dielectric properties, electromagnetic interference shielding and microwave-absorption of short carbon fiber/silica composites. Carbon 2010, 48: 788-796.

[37] Ranjan R, Kumar R, Kumar N, et al. Impedance and electric modulus analysis of Sm-modified $\mathrm{Pb}\left(\mathrm{Zr}_{0.55} \mathrm{Ti}_{0.45}\right)_{1-x / 4} \mathrm{O}_{3}$ ceramics. J Alloys Compd 2011, 509: 6388-6394.

[38] Abo El Ata AM, El Hiti MA, El Nimr MK. Room temperature electric and dielectric properties of polycrystalline $\mathrm{BaCo}_{2 x} \mathrm{Zn}_{x} \mathrm{Fe}_{12-2 x} \mathrm{O}_{19}$. J Mater Sci Lett 1998, 17: 409-413.

[39] Dutta S, Choudhary RNP, Sinha PK. Impedance spectroscopy studies on $\mathrm{Fe}^{3+}$ ion modified PLZT ceramics. Ceram Int 2007, 33: 13-20. 